\title{
Anatomía de los tendones extensores del quinto dedo de la mano
}

\author{
H. F BIANCHI \\ 3Ra CÁtedra de ANATOMía. FaCULTad DE MEDicina UBA. \\ CÁTEDRA ANATOMÍA FUNDACIÓN HA BARCELÓ. \\ Correspondencia: \\ 3ra Cátedra de Anatomía \\ Facultad de Medicina UBA \\ Facultad de Medicina Fundación HA Barceló. \\ Buenos Aires. Argentina. \\ e-mail: hbianchi@fmed.uba.ar
}

En este trabajo se disecaron 40 cadáveres con el fin de aclarar la anatomía distal de los extensores que actúan en el 5을 dedo y su papel en el signo de Wartenberg.

Palabras clave: Tendones extensors, anatomía.
In this paper we dissect 40 cadavera aiming to clarify the distal insertions of the extensor of the small finger, and the role of each structure on Wartenberg's sign.

Key words: Extensor tendons, anatomy.

Rev. Iberam. Cir. Mano - Vol. 33 • Núm. 67 • Noviembre 2005 (44-48)

\section{INTRODUCCIÓN}

L a inserción del extensor propio del meñique $(\mathrm{EDM})^{1}$ en la base de la primera falange (F1), tubérculo medial, y su relación con el signo de Wartenberg ${ }^{1}$ y los desacuerdos con relación a la presencia del extensor común del quinto dedo de la mano (EDC5) en diferentes publicaciones, nos motivó a rever la disposición de estos elementos. La revisión también se extendió a la juntura tendinae entre los tendones mencionados y con el extensor común del cuarto dedo (EDC4).

\section{MATERIAL Y MÉTODO}

En la 3 ${ }^{\mathbf{a}}$ Cátedra de Anatomía Normal de la Facultad de Medicina de la Universidad Nacional de Bs. As. y la Cátedra de Anatomía de la Facultad de Medicina del Instituto Universita- rio de Ciencias de la Salud de la Fundación HA Barceló, se disecó en forma tradicional el aparato extensor del quinto dedo de la mano en 36 cadáveres adultos, sin tener en cuenta el sexo, edad ni posición anatómica. Treinta piezas fueron formolizadas y seis frescas.

Para establecer la presencia del EDC5 se consideró como tal cuando el tendón terminaba directamente o por una de sus lengüetas fusionándose con las del EDM en el dorso de F1, es decir, formando parte del aparato extensor del quinto dedo, lo cual los diferencia de la juntura tendinae que une dos tendones vecinos a nivel metacarpofalángico (MCF), a nivel de la denominada cincha extensora metacarpofalángi$\mathrm{ca}^{3}$. Se analizó si el EDM tomó inserción en el tubérculo medial de la base de F1, para lo cual a la disección clásica se agregó el abrir el tendón a lo largo para mejor visualización. Se realizaron esquemas, se fotografiaron las piezas y se clasificaron los resultados. 


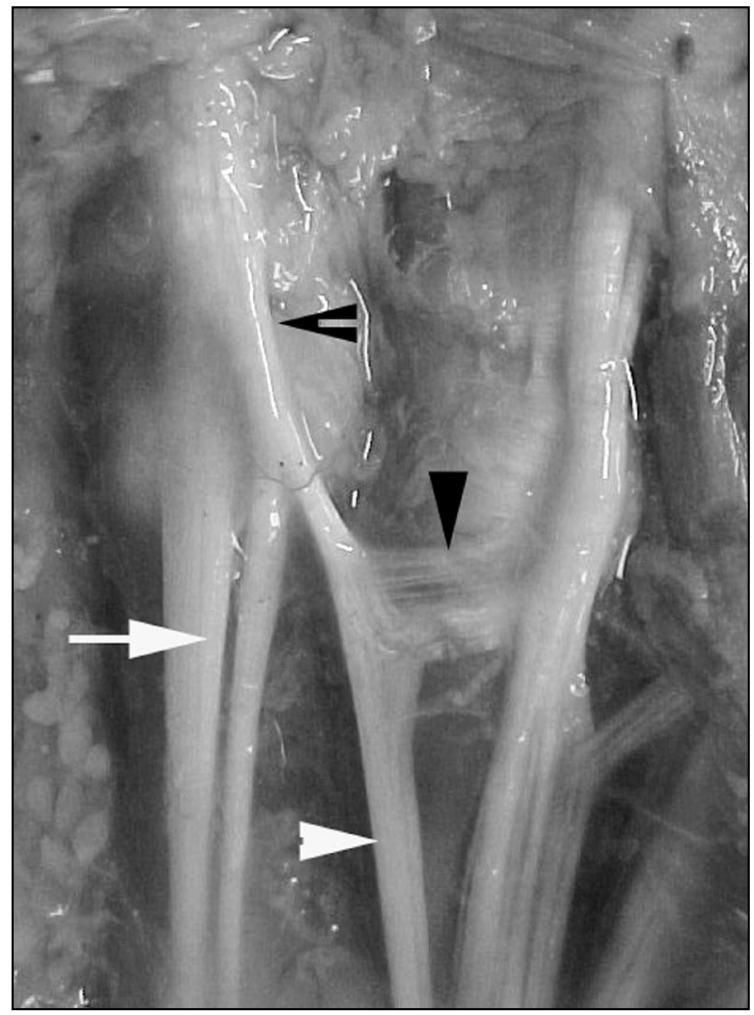

Figura 1. Flecha blanca: EDM. Triángulo blanco: EDC5. Triángulo negro: juntura tendinae. Flecha hendida: fusión del EDC5 con el EDM en dorso MCF.

\section{RESULTADOS}

\section{Grupo I: Extensor Digiti Minimi (EDM)}

\section{a) Numero de tendones}

En todos los casos se encontró un solo cuerpo muscular; en treinta casos $(83,3 \%)$ (Figura 1) se continuó con dos tendones, en tres casos con uno $(8,3 \%)$.

En un caso se observaron tres tendones, dos de la manera habitual el tercero uniéndose al EDC4 a la altura de la articulación MCF.

En tres casos $(8,3 \%)$ observamos desdoblamiento de los tendones en lengüetas que se volvían a unir antes de llegar al nivel MCF: un caso $(2,7 \%)$ por desdoblamiento de las lengüietas radial y cubital, y dos casos por desdoblamiento de la lengüeta radial $(5,4 \%)$.

Fusionados en el dorso de F1 los tendones forman el aparato extensor extrínseco y se disponen de manera habitual.

En ninguna de las preparaciones se pudo observar la inserción del EDM en la base de la F1.

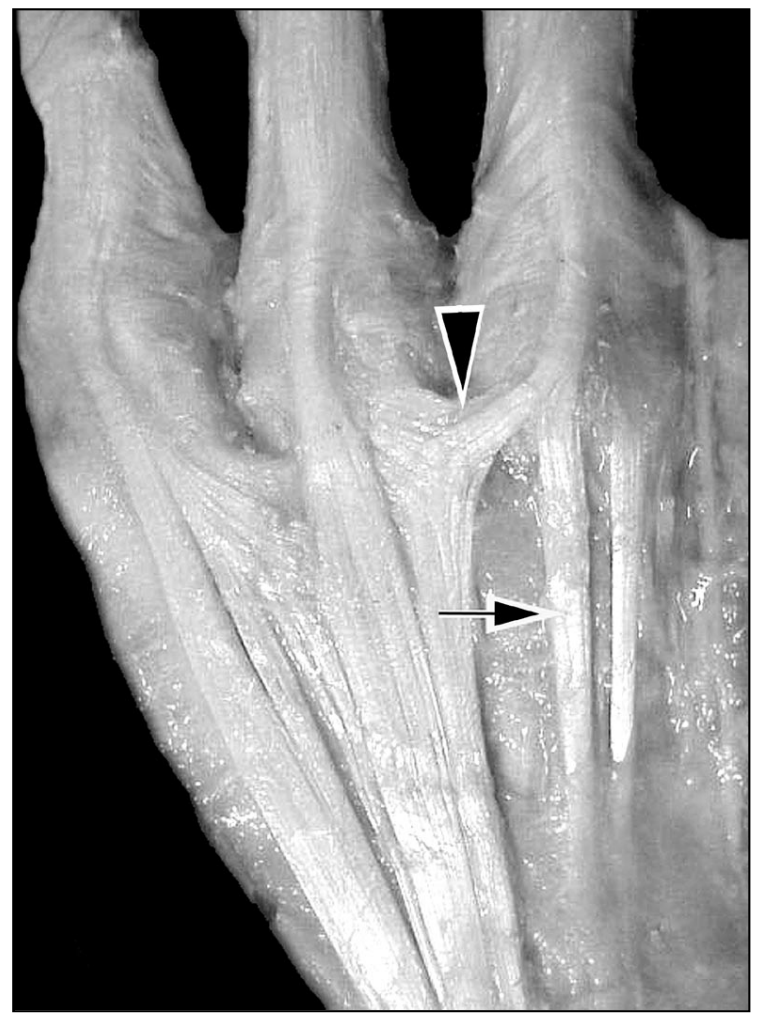

Figura 2. Flecha: EDM. Triángulo: juntura tendinae transversal.

b) Alineación de los tendones de división del EDM con relación al eje del quinto metacarpiano.

En 25 casos $(69,4 \%)$, ambos se disponían alineados. En seis casos (16.6\%), existió desviación del tendón medial hacia cubital (Figura 2), y en cuatro casos $(11,1 \%)$, del tendón lateral hacia radial. En un caso $(2,7 \%)$, ambos tendones se encontraron desviados hacia cubital.

\section{Grupo II: Extensor común del quinto dedo (EDC5).}

El EDC5 no se encontró en 6 casos (16,67\%), (Figura 2).

Se lo observó dividido en un tendón y una juncturae tendinum (JT) en 22 casos $(61,1 \%)$ (Figura 1), dos tendones en 4 casos $(11,1 \%)$ (Figura 3), lo que da un total de presencia de un $72,2 \%$.

Se encontraron 4 casos $(11,1 \%)$ en los que EDC5 se divide en dos JT (Figura 4) que terminan a nivel MCF sobre los tendones del ECD4 y EDM. 


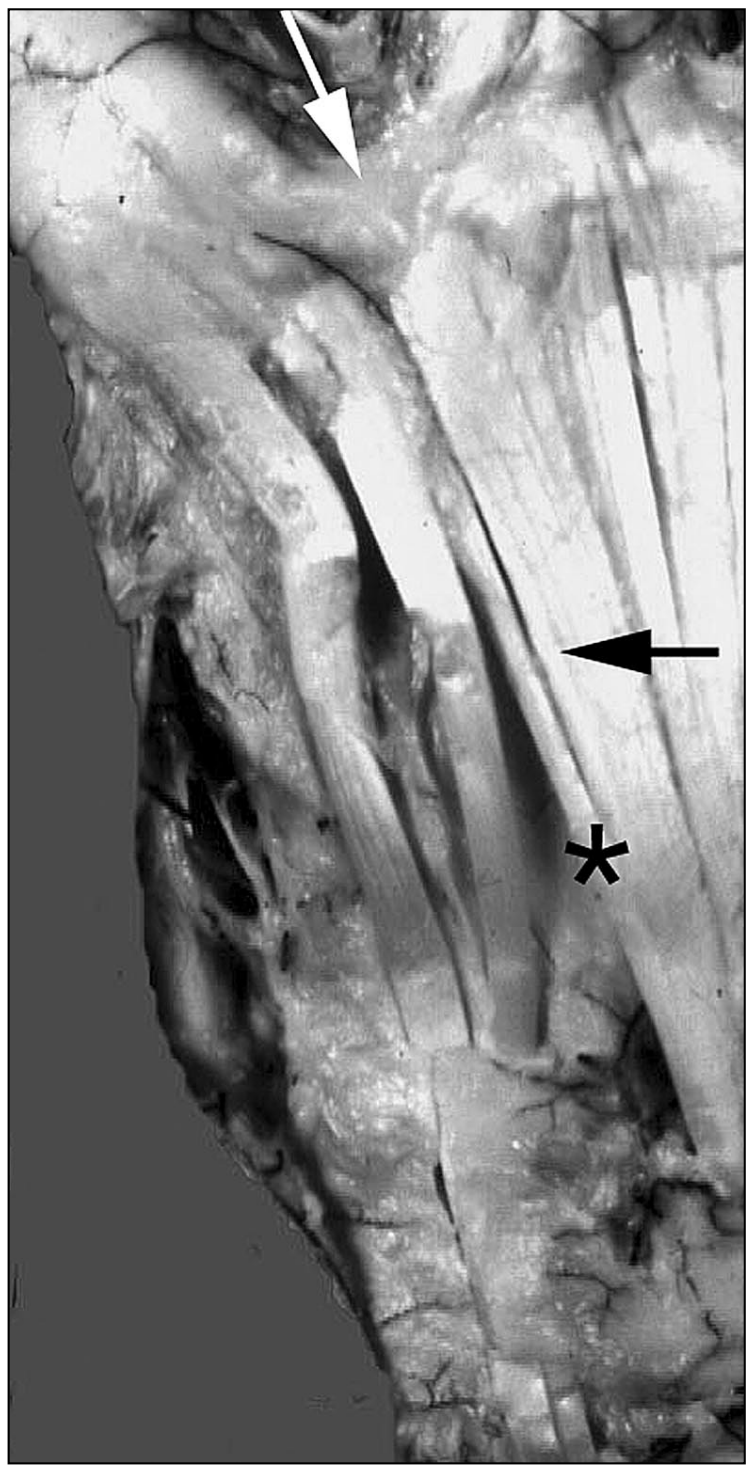

Figura 3. Flecha negra: ECD5. Flecha blanca: división del ECD5 en dos tendones para el EDC4 y EDM. *: tendón accesorio del EDC5 al EDM.

\section{Grupo III: Extensores accesorios.}

Hubo siete casos $(23,3 \%)$ con tendones accesorios al EDC5, y un caso $(3,3 \%)$ con tendón accesorio al EDM. De los accesorios al EDC5, seis terminaron en la lengüeta radial del EDM, próximos a la confluencia con el tendón común principal, mientras que uno lo hizo sobre el EDC5. En dos casos se apreció una lengüeta del tendón del cubital posterior que perfora el techo del sexto compartimiento del retinaculo dorsal del carpo y penetra en la vaina del EDM, al que no se incorpora, llegando a la vertiente lateral de la cabeza del quinto metacarpiano (Figura 5).

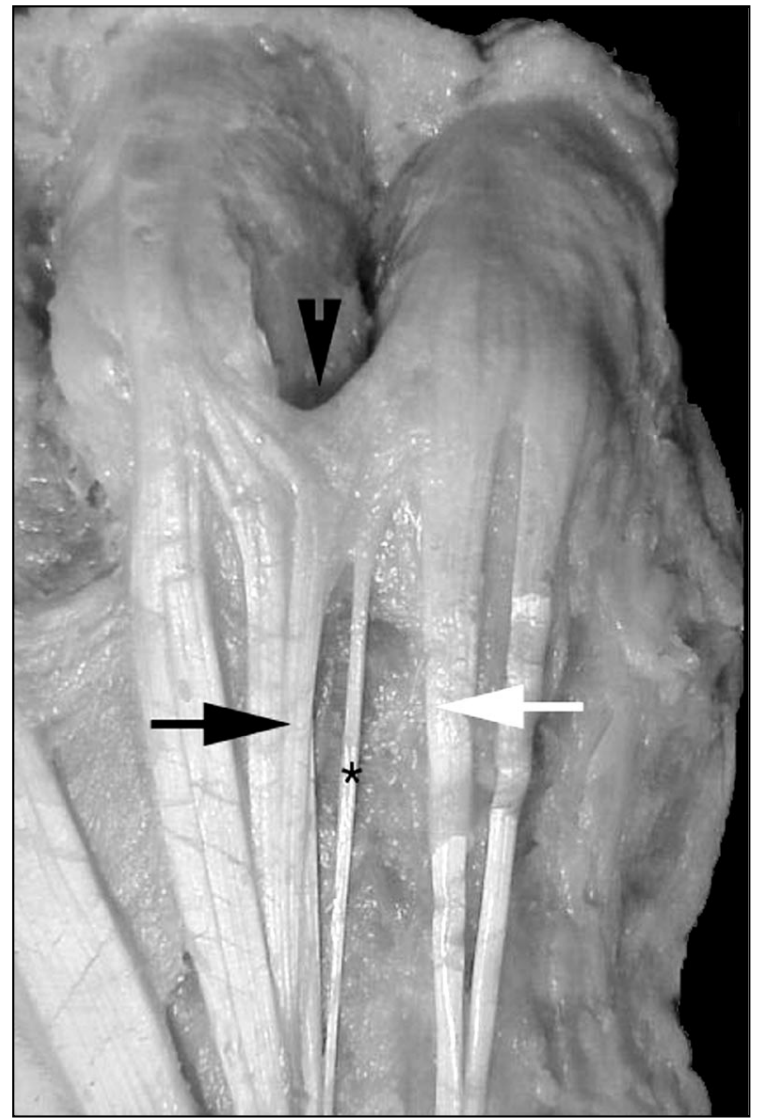

Figura 4. Flecha blanca: ECM. Flecha negra: ECD5. Flecha hendida: juntura tendinum. *: tendón accesorio del EDC5 a la juntura entre el EDC5 y el EDM.

\section{Junctura Tendinum}

La JT entre el EDC5 y el EDC4 fue de tipo tendinoso en 26 casos (72\%) (Figuras 2-4), ligamentario en tres $(8,3 \%)$ (Figura 5) y fascial en uno $(2,7 \%)$ (Figura 4), con dirección oblicua. Cuando el EDC5 no existe, su dirección es transversal (Figura 2) excepto en dos casos de disposición oblicua (Figura 5).

\section{DISCUSIÓN}

Hay acuerdo con todos los autores consulta$\operatorname{dos}^{1-16}$, en que el EDM se divide en un alto porcentaje de casos en dos tendones: uno radial y otro cubital La división tendinosa tuvo lugar distal al quinto compartimiento extensor del carpo, en el que sólo una vez encontramos dos correderas independientes. La presencia de tres tendones con uno terminado en el EDC4 es coincidente con descripciones previas ${ }^{17}$. Es posible 


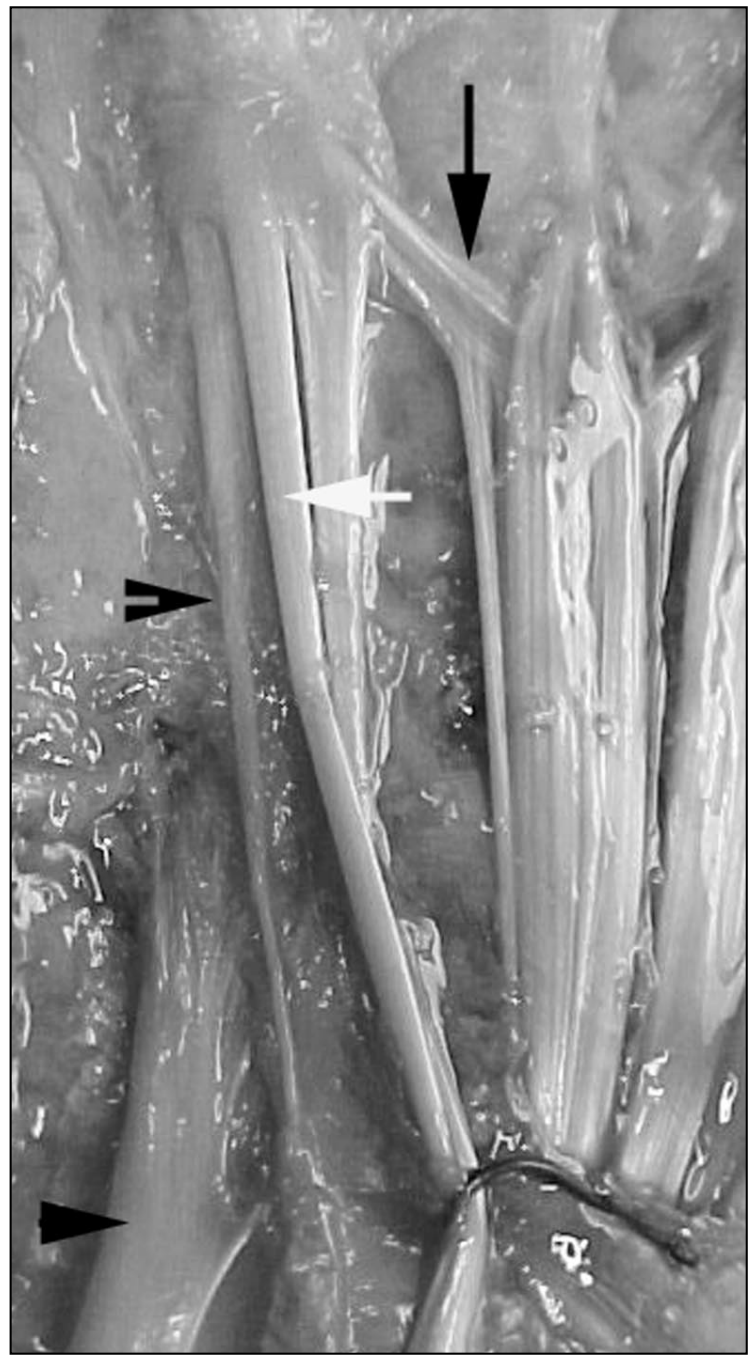

Figura 5. Triángulo negro grande: tendón del cubital posterior. Triángulo negro pequeño: su tendón accesorio que se une al EDM. Flecha blanca: EDM. Flecha negra: JT.

comprobar divisiones en una o dos lengüetas del EDM, que se volvían a unir independientemente de su origen sobre el dorso de la $\mathrm{MCF}^{9}$. Ésta podría corresponder a las estrías o fisuras longitudinales descriptas por Gonzalez ${ }^{1}$. En nuestra serie no encontramos desdoblamiento del cuerpo muscular ni agenesia tendinosa ${ }^{17}$.

La lengüeta radial fue en la mayoría de los casos de mayor entidad, hasta casi duplicar las dimensiones de la lengüeta cubital, lo cual podría deberse a la necesidad de balancear la fuerza de tracción en dirección cubital que por su ubicación tiende a ejercer la lengüeta cubital.

La alineación con el eje del quinto metacarpiano fue la regla en la mayoría de los casos, excepto aquellos con una lengüeta cubital netamente desviada hacia medial. Lo mismo podemos decir de la lengüeta radial cuando estuvo desviada hacia lateral (un solo caso), no coincidiendo con el porcentaje mencionado por Gonzalez ${ }^{1}$.

En lo que se refiere a la inserción de la lengüeta cubital del EDM en el tubérculo de la base de la $\mathrm{F} 1^{1}$, en ninguno de nuestros casos pudimos observarla, coincidiendo con todos los autores consultados, entre ellos Manes y Lima ${ }^{17}$. Los que describen esta inserción ${ }^{1}$ interpretan que su presencia es la base del signo de Wartenberg observado en las lesiones traumáticas del nervio cubital, por lo cual se plantea la hipótesis del desbalance extrínseco intrínseco.

En relación con la discutida presencia del EDC5, estamos de acuerdo en que la diferencia en las cifras de los autores consultados, entre 30 y $80 \%$ se debe y aquí coincidimos con Schroeder $^{10}$, en que las discrepancias son debidas a disparidad de criterios al considerar que es tendón, lengüeta o JT, ya que hay quien considera como JT lo que otros consideran tendón ó lengüeta tendinosa. Hay quien niega su existencia ${ }^{21}$.

En nuestra revisión el EDC5 configuró una estructura anatómica individual separada claramente del EDC4, de quien algunos autores lo consideraran una derivación ${ }^{14-15}$. La disposición anatómica habitual es la de un tendón que se divide en un tendón que se une al EDM y en una JT para el EDC4, siendo mencionadas las otras formas de terminación en dos tendones ${ }^{4}$ $\mathrm{y}$ en $\operatorname{dos} \mathrm{JT}^{14}$.

Por lo expuesto se podría explicar que la baja morbilidad reportada después de una transferencia del $\mathrm{EDM}^{22}$ podría estar en relación con la presencia de un EDC5 en alto porcentaje.

El tipo de JT que une el EDC4 con el EDM cuando el EDC5 está ausente, es de tipo tendinoso y en general de dirección transversa ${ }^{19-22}$.

Los tendones aberrantes toman importancia pues pueden convertirse en estructuras que dificulten por su posición la movilización del tendón del EDM durante una transferencia tendinosa ${ }^{15-18}$.

Ante la no existencia del EDC5, se debería corroborar la presencia de una JT de dirección oblicua la cual podría permitir una cierta extensión del dedo, aunque disminuida en amplitud, 
ya que por su dirección una JT transversa no permite una línea de tracción apropiada que resulte en extensión digital, circunstancia que desaconseja en principio a proceder a la transferencia del EDM.

\section{CONCLUSIONES}

De lo expuesto se concluye que el EDC5 existe aunque su presencia es variable según los autores consultados pero que justifica la baja morbilidad observada luego de una transferencia tendinosa del EDM, debiendo asegurarse en su ausencia de la presencia de una JT de dirección oblicua que asegure la línea de tracción exten- sora y evitar un déficit en la extensión digital, ya que la presencia de una JT transversal no asegura tal efecto.

No se encontró la inserción del EDM en el tubérculo falangico por lo cual la presencia del signo de Wartenberg entendemos se debe una alteración del balance extrínseco- intrínseco al nivel de la MCF del meñique.

Los tendones supernumerarios importan ya que pueden dificultar la movilización del tendón cuando se transfiere.

Es posible que las diferencias entre ausencia presencia del EDC5 continúen porque resulta lógico que ante tanta variante morfológica los investigadores las interpreten en forma diferente.

\section{BIBLIOGRAFÍA}

1. Gonzalez, M. H.; Gray, T., Ortinau, E.; Weinzweig, N.: The extensor tendon of the little finger: An anatomic study. J. Hand Surg 1995; 20-A: 844847.

2. Beannis, H.; Bouchard, A.: Nouveaux éléments d'anatomie descriptive et d'embryologie. Baillière et Fils. Paris 1894.

3. Zancolli, E. A.; Cozzi, E.: Atlas de anatomía quirúrgica de la mano ed Panamericana Madrid 1992

4. El-Badawi, M. G., Butt, M. M.; al Zuhair, A. G.; Fadel, R. A.: Extensor tendons of the fingers: arrangement and variations II Clin Anat. 1995; 8: 391 398.

5. Fort, J. A.: Anatomie descriptive et dissection. Paris Octave Edition: 1892.

6. Poitevin, L.; Abásolo, E.: Extensor propio del meñique. Investigación sobre su variabilidad. Estudio de 50 casos. Publicaciones de la Sociedad Argentina de Anatomía, 1987.

7. Poirier et Charpy: Traité d'anatomie humaine. Tomo 2 fascículo 1. Masson editores, $\mathrm{Pa}-$ ris, 1912.

8. Poitevin, L.; Bianchi, H.; Valente, S.: Variaciones del aparato extensor extrínseco del quinto dedo de la mano. Publicacio- nes de la Asociación Argentina de Anatomía, 1996.

9. Sappey Ph, C.: Traité d'anatomie descriptive Delahaye editores, Paris, 1876.

10. Schroeder von, H. P.; La Jolla, C. A.; Botte, J.: Anatomy of the extensor tendons of the fingers: variation and multiplicity. J. Hand Surg 1995; 20A: 2734.

11. Tubiana, R.: Traité de chirugie de la main. Masson ed., Paris, 1980.

12. Hirai, Y.; Yoshida, K.; Yamanaka, K.; Inoue, A.; Yamaki, K.; Yohsizuk, M.: Anatomic study of the extensor tendons of human hand. J. Hand Surg 1996; 26A: 1009-1015.

13. Godwin, Y.; Ellis, H.: Distribution of the Extensor Tendons on the dorsum of the Hand, Clin Anat 1992; 5: 394-403.

14. Zilber, S.; Oberlin, C.: Anatomical variations of the extensor tendons to the fingers over the dorsum of the hand. A study of 50 hands and a review of the literature. Plast Reconstr Surg 2004; 113: 214-221.

15. Seradge, H.; Tian, W.; Roberts, C.: Clinical significance of an extensor tendon anomaly to the little finger. A new finding, J. Okla State Med Assoc 1999; 92:7-9.
16. Testut, L.: Anomalies Musculaires chez l'homme. Masson ed., Paris, 1884.

17. Manes, L.:, Lima, R.: Relaciones anatomo funcionales entre los músculos extensores y el abductor del quinto dedo. Publicaciones de la Sociedad Argentina de Anatomía, 1978.

18. Schoroeder von, H. P.; Botte, M.: The functional significance of the long extensors and juncturae tendinum in finger extension: J. Hand Surg 1993; 18A: 41-47.

19. Schroeder von, H. P.; Botte, M.; Gellman, H.: Anatomy of the junturae tendinum of the hand, J. Hand Surg 1990; 15-A: 595602.

20. Wehbe, M. A.: Junctura Anatomy. J. Hand Surg 1992: 17A: 1124-1129.

21. Robinson Hagáis, M. K.; Halperin, N.: Restoration of pinch in ulnar nerve palsy by transfer of split extensor digiti mini$\mathrm{mi}$ and extensor indicis. J. Hand Surg 1992; 17: 622-624.

22. Pearl, R. M.; Hentz, V. R.: Extensor digiti minimi transfer to prevent recurrent ulnar drift. Plast Reconstr Surg 1993; 92: 507-10.

23. Voche P, Merle M. Wartenber$g$ 's sign. A new method of surgical correction. J. Hand Surg 1995; 20-B: 49-52. 